\title{
La formation à distance aux États-Unis
}

L'opinion des enseignants du supérieur

Distance Learning in the United States. The opinion of university professors

Formación a distancia en Estados Unidos. La opinión de profesores universitarios

Jim Perley

\section{OpenEdition}

\section{Journals}

Édition électronique

URL : http://journals.openedition.org/ries/2769

DOI : 10.4000/ries.2769

ISSN : 2261-4265

Éditeur

Centre international d'études pédagogiques

\section{Édition imprimée}

Date de publication : 23 septembre 1999

Pagination : 135-140

ISSN : $1254-4590$

\section{Référence électronique}

Jim Perley, "La formation à distance aux États-Unis », Revue internationale d'éducation de Sèvres [En

ligne], 23 | 1999, mis en ligne le 23 septembre 2002, consulté le 14 novembre 2019. URL : http://

journals.openedition.org/ries/2769; DOI : 10.4000/ries.2769 


\title{
La formation à distance aux États-Unis ${ }^{1}$
}

\section{L'opinion des enseignants du supérieur}

\author{
Jim Perley
}

\section{Distance Learning in the United States. The opinion of university professors}

Universities as guarantors of academic and educational quality in higher education are confronted with the consequences of the development of ICT, the vector of increasingly wide information, a modern tool serving learning, but also the source of profits through the appearance of on-line courses. In the United States, an association of university professors has started a battle ... for quality

Formación a distancia en Estados Unidos. La opinión de profesores universitarios

La universidad, garante de la calidad académica y pedagógica de la enseñanza superior, se encuentra confrontada a las consecuencias del desarrollo de las tecnologías de información y comunicación, vectores de una más amplia información, instrumentos modernos al servicio de la formación y también fuente de ganancias con la llegada de los cursos en línea. En Estados Unidos, una asociación de universitarios combate...por la calidad.

La formation à distance, diffusée à l'aide d'ordinateurs, aura certainement sur l'enseignement supérieur un effet aussi important que l'apparition de l'imprimerie. De nouveaux moyens de transmettre un enseignement seront disponibles, les structures et les contenus des cours changeront, une nouvelle pédagogie va se développer, et de nouvelles possibilités d'atteindre des catégories d'étudiants jusqu'alors isolés vont voir le jour. Les moyens par lesquels l'information pourra être transmise vont se multiplier sous des formes que l'on n'a pas encore imaginées.

1 Cet article a été traduit par Jean-Paul Gratias. 
L'Association américaine des professeurs d'université ${ }^{2}$ est une organisation professionnelle d'enseignants d'université et de membres de diverses facultés. Notre association a été fondée en 1915 en raison des préoccupations que suscitaient la question de la liberté d'action des universitaires et le problème de la stabilité de leur emploi. Depuis sa création, nous avons élaboré et diffusé des protocoles judicieux propres à l'institution universitaire, et qui concernent aussi bien les politiques pédagogiques, les procédures à employer - par exemple, pour le choix et la nomination d'un titulaire de chaire ou son licenciement pour motif grave - que les problèmes spécifiques aux femmes qui enseignent à l'université et les critères d'accréditation à utiliser pour déterminer la valeur universitaire des institutions. Il ne s'agit que de quelques exemples des domaines dans lesquels nous avons travaillé. Il nous est arrivé d'avoir à exprimer, devant un tribunal, nos positions sur des problèmes concernant notre profession. Nous avons collaboré avec des organisations dont les membres appartenaient à des conseils d'administration d'institutions universitaires, afin de définir les critères propres à la direction des établissements d'enseignement supérieur.

En bref, au cours des années, nous avons toujours été extrêmement attentifs aux caractéristiques de l'enseignement supérieur qui en garantissent la qualité. Cet intérêt ne s'est jamais démenti, et il est bien présent dans notre étude du développement et de l'accréditation des institutions qui ne dispensent leur enseignement qu'en ligne.

Cette année, nous avons assisté en mars à la création - et à l'accréditation par la North Central Accreditation Agency - de la Jones International University, une université qui fonctionne entièrement en ligne, et dont les quartiers généraux sont situés à Englewood, au Colorado. Plus récemment encore, l'éditeur de manuels Harcourt-Brace a annoncé son projet d'ouvrir à la fois un lycée et un établissement d'enseignement supérieur en ligne.

En présentant le projet de cette nouvelle université, son directeur affirme qu' " un marché existe ". Dans ses fonctions précédentes de recteur de l'enseignement supérieur du Massachusetts, il militait pour la suppression des postes de titulaires à durée illimitée et pour l'application de mesures telles que la réduction des effectifs, courantes dans le monde de l'entreprise. En réponse à ce projet, Charles Crook, président de la New England Association of Schoolsands Colleges - l'instance qui examinera la demande d'accréditation de cette nouvelle université - est réputé avoir déclaré qu'il fallait (sur place) des responsables qualifiés pour contrôler le programme universitaire, ajoutant qu' « ils n'appartiendront pas nécessairement à la faculté ".

Quels genres d'inquiétudes suscitent ces orientations pour les membres de la faculté ? Nos appréhensions portent sur des questions de qualité et de méthodes et concernent les critères utilisés pour accorder une accréditation.

2 American Association of University Professors (AAUP). 
À un niveau plus fondamental, les principes que nous défendons visent à préserver les caractéristiques de l'enseignement supérieur qui, historiquement, ont servi la société en formant des diplômés capables d'analyser l'information, de découvrir de nouvelles connections entre les différents apprentissages et de déterminer, en faisant preuve d'esprit critique, les meilleures approches pour développer de nouvelles façons d'utiliser ces synthèses afin d'analyser l'inconnu.

Nous pensons que c'est l'université, avec sa longue expérience des diverses disciplines, qui comprend le mieux les buts et les objectifs de l'enseignement. Bien que la structure d'un cours puisse être le produit de plusieurs individus travaillant ensemble, le véritable enseignement dans la salle de cours doit laisser la possibilité d'adapter les supports pédagogiques aux étudiants, de les façonner à partir de leur capacité de compréhension, de prêter attention aux différences d'appréhension de l'enseignement dues à la diversité des origines socioculturelles et de modifier au besoin la pédagogie une fois le cours commencé, pour répondre utilement aux besoins des étudiants.

Aucun programme automatisé, dicté par un comité, et décomposé en plusieurs thèmes assortis d'un minutage, ne peut reproduire ce qui se passe dans la dynamique d'une salle de cours. Il est difficile, sinon impossible, de concevoir comment un cours d'expression en public, par exemple, pourrait être proposé en ligne sans le bénéfice des réactions du public et de l'interaction immédiate de l'orateur.

Aucun cours de sciences ne peut être dispensé sans application pratique dans un laboratoire équipé. Il est difficile d'imaginer comment des connaissances peuvent être transmises sans la présence d'un enseignant attentif, à l'écoute de ses élèves, et fort de l'expérience que tous les membres de l'université ont pu acquérir dans une salle de cours. C'est un fait établi qu'aucun programme d'enseignement automatisé ne peut reconnaître le moment où le stade de la compréhension véritable est atteint et où les étudiants signalent qu'il est temps d'approfondir.

Notre préoccupation s'aggrave lorsque des institutions entières sont envisagées qui ne compteraient que deux professeurs à plein temps. La conception selon laquelle une université ou un établissement d'enseignement supérieur rassemble des administrateurs, qui ont leurs racines au sein de la faculté, et des étudiants, qui travaillent avec leurs professeurs pour que les savoirs se transmettent et progressent, est complètement battue en brèche par l'idée d'une institution composée uniquement d'administrateurs engagés pour gérer des programmes et faire tourner des ordinateurs, sans corps enseignant.

Une autre source d'inquiétude grave pour les enseignants est l'apparition d'un nouveau type d'administrateurs, souvent impliqués dans l'élaboration d'un « enseignement supérieur » en ligne, qui ne sont pas des professeurs formés dans une discipline mais des gestionnaires du supérieur. En fait, nombre de ces nouveaux administrateurs possèdent des diplômes de "gestion éducative », et il 
n'est pas facile de savoir si leur priorité est de recruter des étudiants ou de fournir un enseignement de qualité. Ces gestionnaires semblent avoir plus de points communs avec les PDG des grandes sociétés modernes qu'avec les administrateurs dont l'objectif majeur est de permettre à l'enseignement d'exister. Les profits passent avant tout, comme à l'université de Phoenix, et les besoins du marché deviennent la seule raison d'exister. Si un domaine d'étude ne trouve pas de clients, on le considère comme inutile. Peut-on imaginer des études supérieures sans sciences physiques et sans poésie ? Une personne véritablement cultivée peut-elle être produite par le programme d'études parcellaires qui résulterait de la mise en application d'un tel consumérisme?

Pour nous, à l'AAUP, la recherche est un secteur vital de l'enseignement supérieur. Quel que soit le niveau d'études considéré, repousser les frontières de l'ignorance est une nécessité dans ce que nous appelons l'enseignement "supérieur ", c'est l'élément majeur qui fait la distinction entre l'université et le secondaire. Il est difficile d'imaginer un cours, même dans le domaine technique, dans lequel les problèmes existants ne seraient pas analysés à la lumière des connaissances acquises. Concevoir de nouvelles approches pour la résolution de problèmes, examiner les problèmes selon un point de vue interdisciplinaire, c'est ce que permet un rassemblement d'intelligences travaillant en commun. Les conversations avec les étudiants dans la salle de cours, les contacts informels avec les collègues au déjeuner font souvent jaillir des idées nouvelles et inattendues.

138 Des possibilités d'interactions aussi importantes sont réduites ou inexistantes lorsque l'enseignement est diffusé en ligne. Il est impossible d'imaginer que le type d'interactions et le foisonnement d'idées qui naissent dans la salle de cours et les couloirs puissent se produire dans un forum de discussions ou dans un échange de courriers électroniques. La liberté de se conduire en irresponsable dans une discussion est restreinte par la nécessité de rendre des comptes qui n'est concevable que dans une atmosphère de véritable face-à-face entre êtres humains. C'est dans ce genre d'arène où le débat civique peut survenir à tout moment et où les idées s'affinent grâce à l'interaction entre interlocuteurs - et non pas dans la diatribe virulente - que l'on voit la véritable valeur de la salle de cours.

Nous craignons que l'enseignement en ligne ne détruise l'idée que l'éducation naît dans un milieu d'érudits. Et nous croyons fermement que l'existence d'une telle communauté d'érudits - professeurs et étudiants - entre lesquels se produisent des échanges, est exactement la définition de ce que nous sommes.

À un niveau beaucoup plus large, ces nouveaux projets soulèvent des questions fondamentales sur la véritable nature de l'enseignement supérieur. La plupart des professeurs de faculté n'hésiteraient pas longtemps avant d'affirmer que le plus important dans ce qu'ils font ce n'est pas l'information transmise à l'étudiant, même si c'est elle qui fournit la matière des questions posées aux exa- 
mens. Nous ajouterions tout aussi vite que l'accès à la technologie est une nécessité de plus en plus importante pour les professeurs de faculté qui veulent élaborer des programmes d'études et accéder à l'information. Mais nous pensons sincèrement que ce qui se passe dans la salle de cours, dans le laboratoire, dans l'amphithéâtre et sur le terrain est beaucoup plus subtil et durable. Notre travail consiste à développer les esprits, à inculquer l'idée qu'il est nécessaire d'apprendre tout au long de sa vie, à faire mûrir des citoyens informés qui réfléchissent. En bref, nous faisons bien autre chose que simplement transmettre à des individus les compétences nécessaires pour amasser l'information. Notre objectif consiste à former la prochaine génération de dirigeants, des dirigeants capables de repérer les interconnections et de faire progresser la société.

L'enseignement supérieur a bien servi la société. L'enseignement supérieur américain, en particulier, a la réputation d'avoir produit pour la société, aussi bien au niveau international que national, des diplômés compétents. Il a rencontré le succès parce qu'il a su ouvrir les portes de chaque discipline et de chaque domaine universitaire à des esprits critiques et ne s'est pas contenté de produire des étudiants purs clones de leurs professeurs. Il a engendré l'excellence parce qu'il a conservé le principe de la liberté d'action des universitaires, la conception de l'administration collégiale, et parce qu'il est resté fidèle à sa conviction que les professeurs d'université sont au cœur de l'entreprise.

Les universitaires ne sont pas des Luddites ${ }^{3}$. Mon utilisation des ordinateurs et des ressources en ligne s'est considérablement accrue depuis cinq ans, et c'est aussi le cas de la vaste majorité de mes collègues. En fait, je suis incapable d'imaginer un retour à un enseignement privé d'accès à l'information en ligne. Mais je suis conscient du fait qu'une part importante des documents accessibles en ligne ne sont ni corrigés ni vérifiés. C'est dans l'apprentissage de l'utilisation correcte de cette information et dans l'appréhension de ses limites que se fait la différence entre un individu qui est simplement formé et un autre qui est véritablement éduqué.

Dans la mesure où les avancées technologiques servent à faire progresser les objectifs des véritables universitaires, capables de choisir leurs méthodes pédagogiques et le contenu de leur enseignement, ces progrès seront utilisés comme des outils d'une importance vitale. Si la technologie est perçue par ceux qui ne sont pas des universitaires à temps plein, comme un moyen de faire des profits plutôt que de produire un enseignement de qualité, elle échouera lamentablement - tout comme, dans les années soixante, l'approche « innovante » de l'enseignement des sciences expérimentales qui a remplacé par des écrans de télévision la véritable utilisation d'un laboratoire.

3 Allusion aux émeutes de 1811-1813 en Grande-Bretagne, au cours desquelles des chômeurs ont détruit les nouvelles machines textiles, jugées responsables de leur sort (NDT). 
Même au sein d'un marché orienté vers le consommateur, tel que celui envisagé par les concepteurs de la Western Governor's University, les étudiants finissent par faire des choix judicieux. Seuls dix étudiants se sont présentés la première fois qu'il a été possible de s'y inscrire pour préparer un diplôme. Ils préfèrent la qualité. Les universitaires aussi.

Si la formation à distance fait progresser les critères considérés comme importants par la profession, si elle permet d'enrichir les contenus et d'élaborer de nouvelles façons d'enseigner, elle sera adoptée par les universitaires désireux de travailler encore plus efficacement avec leurs étudiants. Si elle se contente de proposer une solution de rechange à la faculté et rien d'autre, elle sera rejetée par les gens à la recherche d'une véritable éducation.

À l'AAUP, nous avons le sentiment qu'il est de notre devoir de demander que des modèles d'excellence soient utilisés par les instances d'accréditation et par les personnes chargées de déterminer les critères dans ce qui est appelé l'enseignement supérieur, sans quoi la société et les étudiants risquent d'être dupés. 